\title{
Neutron-deuteron analyzing power data at 19.0 MeV
}

\author{
G. J. Weisel, ${ }^{*}$ W. Tornow, B. J. Crowe III, ${ }^{\dagger}$ A. S. Crowell, J. H. Esterline, C. R. Howell, J. H. Kelley, R. A. Macri, ${ }^{\ddagger}$ \\ R. S. Pedroni,$^{\S}$ and R. L. Walter \\ Department of Physics, Duke University, Durham, North Carolina 27708, USA \\ and Triangle Universities Nuclear Laboratory, Durham, North Carolina 27708, USA \\ H. Witała \\ Institute of Physics, Jagellonian University, Cracow, Poland \\ (Received 21 December 2009; published 23 February 2010)
}

\begin{abstract}
Measurements of neutron-deuteron $(n-d)$ analyzing power $A_{y}(\theta)$ at $E_{n}=19.0 \mathrm{MeV}$ are reported at 16 angles from $\theta_{\text {c.m. }}=46.7$ to $152.0^{\circ}$. The objective of the experiment is to better characterize the discrepancies between $n$ - $d$ data and the predictions of three-nucleon calculations for neutron energies above $16.0 \mathrm{MeV}$. The experiment used a shielded neutron source, which produced polarized neutrons via the ${ }^{2} \mathrm{H}(\vec{d}, \vec{n})^{3} \mathrm{He}$ reaction, a deuterated liquid scintillator center detector (CD) and liquid-scintillator neutron side detectors. A coincidence between the CD and the side detectors isolated the elastic-scattering events. The CD pulse height spectrum associated with each side detector was sorted by using pulse-shape discrimination, time-of-flight techniques, and by removing accidental coincidences. A Monte Carlo computer simulation of the experiment accounted for effects due to finite geometry, multiple scattering, and CD edge effects. The resulting high-precision data (with absolute uncertainties ranging from 0.0022 to 0.0132 ) have a somewhat lower discrepancy with the predictions of three-body calculations, as compared to those found at lower energies.
\end{abstract}

DOI: 10.1103/PhysRevC.81.024003

PACS number(s): 25.10.+s, 24.70.+s, 25.40.Dn

\section{INTRODUCTION}

During the last 15 years, substantial progress has been made on the theoretical description of three-nucleon $(3 \mathrm{~N})$ systems. The improved quality of nucleon-nucleon $(N N)$ potentials, such as Nijmegen [1], Argonne V18 (AV18) [2], and CD-Bonn [3], has resulted in an excellent description of the available $N N$ data. At the same time, improvements were made to three-body calculations based on the $N N$ potential models. In addition to giving reliable predictions of the observables of $3 \mathrm{~N}$ systems, the new three-body calculations make it possible to construct models of possible three-nucleon forces (3NF's) in the nuclear Hamiltonian [4,5]. These theoretical advances call for $3 \mathrm{~N}$ data of adequate precision that will place relatively strong constraints on the nuclear Hamiltonian.

The present article addresses the lack of high-precision neutron-deuteron $(n-d)$ analyzing power data in the energy region between 16 and $40 \mathrm{MeV}$. The gap has been a matter of concern in answering the so-called "three nucleon analyzing power puzzle" (3NAPP) as discussed in Ref. [6]. The 3NAPP refers to the fact that the calculated magnitude for the analyzing power $A_{y}(\theta)$ in polarized neutron and polarized proton elastic scattering from deuterons is significantly lower

\footnotetext{
*Permanent address: Department of Physics, Penn State Altoona, Altoona, Pennsylvania 16601.

${ }^{\dagger}$ Permanent address: North Carolina Central University, Durham, North Carolina 27707.

${ }^{\ddagger}$ Permanent address: Lawrence Livermore National Laboratory, Livermore, California 94550.

§Permanent address: North Carolina A\&T University, Greensboro, North Carolina 27411.
}

than the experimentally observed magnitude. It also refers to the discrepancy between the calculated and experimentally observed magnitude for the deuteron vector analyzing power $i T_{11}(\theta)$ in polarized deuteron elastic scattering from protons.

Table I lists the available high-precision $n-d A_{y}(\theta)$ data below $40 \mathrm{MeV}$. The angular distributions of each data set show a significant discrepancy with predictions based on the high-precision $N N$ potentials referred to previously and the Faddeev method of solving the three-body system. This is especially evident at the maximum of $A_{y}(\theta)$ at the back angles. A useful way to gauge this discrepancy is to calculate the relative difference, that is, the difference between theory and experiment divided by the experimental value at the maximum of $A_{y}(\theta)$. Measured in this way, the discrepancy is about $25 \%$ (experiment is higher) for all neutron energies from 1.2 to $16 \mathrm{MeV}$.

Six years ago, the relative difference of $A_{y}(\theta)$ from $E_{n}=$ 1.2 to $10 \mathrm{MeV}$ showed a different energy trend for $p$ - $d$ scattering than it did for $n-d$ scattering. Experimental work at low energies suggested that this difference was due to the fact that three-body calculations did not include the MottSchwinger interaction between the magnetic moment of the incoming nucleon and the Coulomb field of the deuteron [7]. Three-body calculations incorporating the Mott-Schwinger interaction confirmed this speculation, putting the difference between theory and experiment at the maximum of $A_{y}(\theta)$ for both $p-d$ and $n-d$ scattering at the same level, about $25 \%$ for all energies up to $16 \mathrm{MeV}[17,18]$.

Explaining the 3NAPP has proven to be a difficult challenge. One proposed explanation was that the ${ }^{3} P_{J}$ phase shifts are incorrect, leading to an improper determination of the $N N$ force components in these partial waves [19]. For a short time, chiral perturbation theory seemed to confirm this; 
TABLE I. Available high-precision $n$ - $d$ $A_{y}(\theta)$ data below $E_{n}=40 \mathrm{MeV}$.

\begin{tabular}{lc}
\hline \hline Energy $(\mathrm{MeV})$ & Reference \\
\hline 1.2 & Neidel [7] \\
1.9 & Neidel [7] \\
3.0 & McAninch [8] \\
5.0 & Tornow [9] \\
6.5 & Tornow [9] \\
8.5 & Tornow [9] \\
8.5 & Tornow [10] \\
10.0 & Tornow [11] \\
12.0 & Howell [12] \\
13.0 & Cub [13] \\
14.1 & Tornow [14] \\
16.0 & Fujita [15] \\
30.0 & Dobiasch [16] \\
\hline \hline
\end{tabular}

calculations in the next-to-leading order (NLO) suggested that the $N N$ chiral force will solve the 3 NAPP via modified ${ }^{3} P_{J}$ phase shifts [20]. However, it soon became clear that the ${ }^{3} P_{J}$ phase shifts cannot be improved such that they fit all of the existing $2 \mathrm{~N}$ and $3 \mathrm{~N}$ data. To make matters worse, inclusion of relativistic effects (Wigner rotation of the spin states) into the three-body calculations slightly worsened the discrepancy between theory and experiment [21].

Therefore, theorists turned their attention to another possible explanation of the 3NAPP: the action of three-body forces (3NF's). Initial calculations along these lines have not been promising. Traditional 3NF's, such as the Urbana IX $3 \mathrm{NF}$ or the $2 \pi$-exchange Tuscon-Melbourne $3 \mathrm{NF}$, act over relatively short ranges. When they were included in three-body calculations they influenced $A_{y}(\theta)$ only slightly [4].

Chiral perturbation theory at higher order than NLO offers considerable promise for investigations into 3NF's. Three-nucleon force mechanisms appear for the first time in next-to-next-to-leading order (N2LO). However, calculations at N2LO reproduced the experimentally determined ${ }^{3} P_{J}$ phase shifts correctly and also had only a slight effect on $A_{y}(\theta)$ predictions. Therefore, N2LO cannot eliminate the 3NAPP [22,23]. We still await the difficult calculations of $n$ - $d$ scattering at N3LO, which will also include relatively long-range 3NF mechanisms.

Determining the magnitude of the 3NAPP and its energy dependence above $16 \mathrm{MeV}$ may provide a clue regarding the cause of the 3NAPP (as it did at lower energies in uncovering the importance of the Mott-Schwinger interaction). At the very least, it will provide a reliable database for modelers. A recent review of available proton and neutron data confirms that the relative difference for both $p-d$ and $n-d$ analyzing power is about $25 \%$ between 1.2 and $16 \mathrm{MeV}$ [24]. By adopting a measure of the relative difference based on both the minima and maxima in the angular distributions, it is judged in Ref. [24] that this measure of the discrepancy between theory and experiment stays fairly constant at $25 \%$ until about $25 \mathrm{MeV}$.

However, above $16 \mathrm{MeV}$ it is difficult to make strong conclusions about the trend of the relative difference. This is due to two weaknesses of the database available to Ref. [24]: First, much of the data above $16 \mathrm{MeV}$ are of relatively low precision and second there are no high-precision neutron data between 16 and $30 \mathrm{MeV}$. The $n-d A_{y}(\theta)$ data at $16 \mathrm{MeV}$ of Ref. [15] showed an especially large relative difference (based on both the minimum and maximum) of $36 \%$. The $30 \mathrm{MeV}$ data of Ref. [16] demonstrated that there is still a 3NAPP at this energy, but suggested that the relative difference between theory and experiment is significantly less, about $13 \%$. (Available $p-d A_{y}(\theta)$ data at $30 \mathrm{MeV}$ agree within uncertainties, providing a relative difference of 15\%.) Data are available at $35.0 \mathrm{MeV}$ from Ref. [25], but are of inadequate precision even to enable comparison with three-body calculations. Therefore, it is not possible to say at precisely what energy the 3NAPP ceases to be a problem. The present article's $n$ - $d A_{y}(\theta)$ data at $E_{n}=19.0 \mathrm{MeV}$ represent a first effort at addressing the scarcity of data above $16 \mathrm{MeV}$ and at establishing the magnitude and energy trend of the 3NAPP.

\section{EXPERIMENTAL SETUP}

Gathering our data required five different runs of roughly ten days each. The experiment was conducted in a target room based around the Triangle Universities Nuclear Laboratory (TUNL) Shielded Neutron Source, which features a 180-cmthick wall made of concrete, paraffin, iron, steel, and lead (see Fig. 1). Within the wall, at the end of the beam line, was a gas cell used as a neutron-production target, which was $3.0 \mathrm{~cm}$ long and $1.0 \mathrm{~cm}$ in diameter. Polarized deuterons were produced by TUNL's Atomic Beam Polarized Ion Source (ABPIS), accelerated through an FN Tandem accelerator, and deflected by $20^{\circ}$ to the target room (thus determining the beam

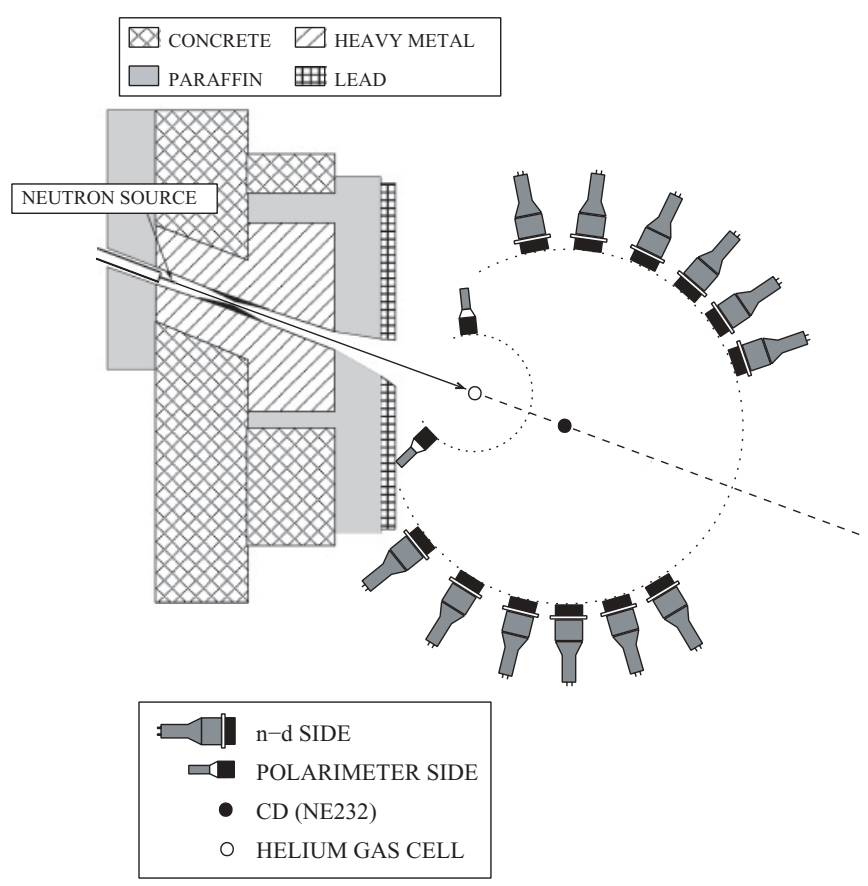

FIG. 1. The experimental setup in the TUNL Shielded Neutron Source Area used to take the present $n-d A_{y}(\theta)$ data at $19.0 \mathrm{MeV}$. 
energy). The gas cell was filled with deuterium to $7.8 \mathrm{~atm}$ and polarized neutrons were produced via the ${ }^{2} \mathrm{H}(\vec{d}, \vec{n})^{3} \mathrm{He}$ source reaction at $0^{\circ}$. The entrance to the gas cell was a $6.35-\mu \mathrm{m}$-thick Havar foil while the exit was a 0.50 -mm-thick gold beam stop. The deuteron beam current reaching the deuterium gas cell ranged typically from 800 to $1200 \mathrm{nA}$. The energy spread in the neutron beam due to the energy loss of the deuteron beam in the deuterium gas and the Havar foil was $\pm 305 \mathrm{keV}$.

The center detector (CD) target was a deuterated liquid scintillator (NE232), encased in a glass cylinder of $4.2 \mathrm{~cm}$ in diameter and $6.4 \mathrm{~cm}$ high. The CD was located $312 \mathrm{~cm}$ downstream from the gas cell and was surrounded by six pairs of neutron side detectors, each of which were located $150 \mathrm{~cm}$ from the center of the $\mathrm{CD}$. A total of 16 angles were measured, covering the $A_{y}(\theta)$ distribution. In each individual run, the six angles were chosen to ensure adequate spacing between side detectors so that cross talk between detectors was minimized. The side detectors used NE213 liquid scintillator and were cylindrical with a diameter of $12.7 \mathrm{~cm}$ and a thickness of either 5.1 or $6.1 \mathrm{~cm}$. Due to the fact that our neutron source was encased by the $180-\mathrm{cm}$-thick wall, the photomultiplier tubes of the $\mathrm{CD}$ and of the polarimeter gas cell were shielded from the direct flux of the neutron source. In the same way, the neutron side detectors were also shielded from the neutron source reaction.

To cancel instrumental asymmetries due to differences in the side detectors and their associated electronics, the ABPIS flips the spin orientation between up and down in relation to the scattering plane at a rate of $10 \mathrm{~Hz}$. The measured asymmetry is defined as

$$
\epsilon(\theta)=\frac{\sqrt{\frac{L_{U} R_{D}}{R_{U} L_{D}}}-1}{\sqrt{\frac{L_{U} R_{D}}{R_{U} L_{D}}}+1},
$$

where $R_{U}$ and $R_{D}$ designate the yields for the right detector when the spin is oriented up and down, respectively (and similarly with $L_{U}$ and $L_{D}$ for the left detector). With these definitions the measured analyzing power is

$$
A_{y}(\theta)=\frac{1}{P_{n}} \epsilon(\theta) .
$$

To monitor the beam polarization $P_{n}$ a polarimeter was constructed using an active, high-pressure gas cell and two neutron side detectors. The gas cell was filled to $100 \mathrm{~atm}$ with a mixture of about $95 \%{ }^{4} \mathrm{He}$ gas and $5 \% \mathrm{Xe}$ gas (added as a wavelength shifter) and had an inner diameter of $4.8 \mathrm{~cm}$. Two photomultiplier tubes viewed the gas cell through two glass windows, one above and one below the beam axis. The centers of the polarimeter side detectors were $36 \mathrm{~cm}$ away from the center of the gas cell and had an area facing the cell of $5.1 \mathrm{~cm}$ wide, $14 \mathrm{~cm}$ high, and a thickness of $7.6 \mathrm{~cm}$. The traditional location for the polarimeter is downstream from the $\mathrm{CD}$. However, due to the geometry of our beam collimation and the length of our CD-side flight paths, we located the polarimeter $80 \mathrm{~cm}$ upstream from the $\mathrm{CD}(232 \mathrm{~cm}$ downstream from the gas cell). This resulted in a small reduction of $n-d$ elastic counts of less than $5 \%$. The polarimeter's side detectors

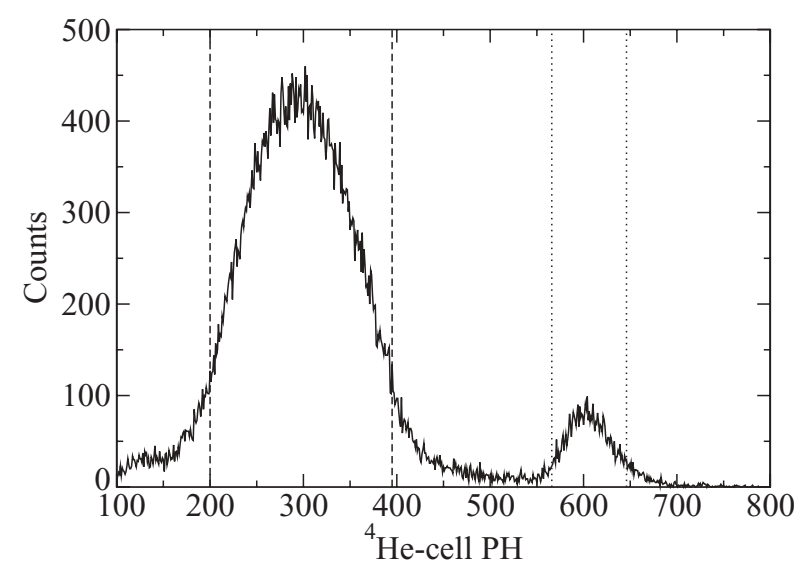

FIG. 2. Pulse height spectrum for the ${ }^{4} \mathrm{He}$ cell. The gate on the right (the dotted vertical lines) was used for the yields of the "elastic neutrons" while the gate on the left (the dashed lines) was used for the "breakup neutrons." See text for explanation.

were mounted at an angle of $\theta_{\mathrm{lab}}=115^{\circ}$, at which the $n+{ }^{4} \mathrm{He}$ analyzing power is large valued and well determined.

To measure the neutron polarization $P_{n}$, we followed a method that is similar to the one described in the next section for the $n-d A_{y}(\theta)$. Here, we only outline the procedure. Pulse-shape discrimination was used on the neutron side detectors to filter the time-of-flight (TOF) spectra. This not only distinguished between the neutron and $\gamma$ pulses but also reduced the accidental background. A gate was then set on the elastic peak in the TOF spectrum and used to filter the pulse height of the ${ }^{4} \mathrm{He}$ cell. When the electronics operated at full capability the resulting pulse-height spectra clearly delineated the peak due to the "elastic neutrons" (the neutrons originating from the ground-state transition of the ${ }^{2} \mathrm{H}(\vec{d}, \vec{n})^{3} \mathrm{He}$ reaction and then scattered from ${ }^{4} \mathrm{He}$ ), as can be seen in Fig. 2. The usual gate used for determining the polarimeter yields appears as the two dotted vertical lines.

However, in the first two of the five ten-day runs, we had trouble with the resolution of the polarimeter. Instead of being clearly deliniated, the elastic neutrons blurred into the lower-energy and far more numerous "breakup neutrons" due to breakup reactions in the deuterium gas cell, such as ${ }^{2} \mathrm{H}(\vec{d}, \vec{n} \mathrm{p})^{2} \mathrm{H}$. Under these circumstances, we did not routinely monitor the elastic neutrons but instead used the breakup neutrons. In this case, the gate used for determining the polarimeter yields was similar to the two dashed vertical lines in Fig. 2. In one way this was a convenient monitor since it allowed us to make a quick determination of relative beam asymmetry. The drawback, of course, is that we needed to translate this result to the asymmetry of the elastic neutrons. To accomplish this, we first isolated the elastic neutrons in a series of special tests by filtering the ${ }^{4} \mathrm{He}$ cell pulse height and the TOF. After finding the asymmetry of the elastic counts using Eq. (1), we calculated the ratio of the asymmetry of the elastic neutrons to the asymmetry of the breakup neutrons. We did this procedure to seven major subdivisions of the first two ten-day runs and four more subdivisions of the later runs (where the elastics were easily isolated as in Fig. 2). We found that the 
ratio was well defined at $0.980 \pm 0.015$. Of course, using this ratio to translate from the asymmetry of the breakups to the asymmetry of the elastics adds an uncertainty to the data for the first two of our five runs.

Once the asymmetry of the elastic neutrons was determined the polarization of the beam was found by using an effective $\mathrm{n}-{ }^{4} \mathrm{He}$ analyzing power. A Monte Carlo simulation modeled single scattering from ${ }^{4} \mathrm{He}$ as well as all relevant double scattering processes involving helium, xenon, iron, and glass. Consideration of the double scattering processes reduced the value taken from Ref. [26] from 0.844 to an effective value of $0.828 \pm 0.017$. The neutron beam polarization $P_{n}$ was computed with Eq. (2). Under normal operation of the ABPIS, the $P_{n}$ rested somewhere between 0.60 and 0.70 but sometimes dipped as low as 0.50 . Significant changes in $P_{n}$ were on the time scale of days; we were careful to separate the data into sequential batches with similar $P_{n}$.

Each event stored by the computer was triggered by a timing coincidence between the $\mathrm{CD}$ and a particular side detector (using a window of width $300 \mathrm{~ns}$ ). The pulse height in the CD was stored using an analog-to-digital converter. In addition, the TOF between the CD and the side detector was stored using a time-to-digital converter. Finally, for each event, information regarding the pulse shape of the side detector signal was stored for the purpose of pulse-shape discrimination. This was done by using a charge-integrating analog-to-digital converter (QDC); one time window integrated the charge in the entire detector pulse and the other window integrated the charge in the leading edge of the pulse. The threshold on the constant fraction device for the $\mathrm{CD}$ was kept as high as possible to lower the computer dead time, but not so high as to cut into the signals associated with the small-angle side detectors. The thresholds of the side detectors varied with the angle, ranging from $2.0 \times{ }^{137} \mathrm{Cs}$ for the forward angles to $0.50 \times{ }^{137} \mathrm{Cs}$ for the back angles. With data being taken for the polarimeter (which had its own trigger) and six detector pairs, the dead time of the data-acquisition system was usually in the range of 10 to $20 \%$.

\section{DATA SORTING}

To identify the desired $n$ - $d$ elastic events, a number of sorting procedures were employed using a data analyzer built on CODA software along with the PAW graphics display. To accomplish pulse-shape discrimination (PSD) for each side detector we created a two-dimensional (2D) histogram that displayed charge in the entire pulse versus charge in the tail only. By drawing a 2D gate around the desired elastic events, we greatly reduced the accidental background and eliminated the events due to $\gamma$ rays (most of which are produced when the deuteron beam hits the gold beam stop at the end of the neutron production cell).

The use of the QDC's worked adequately but were subject to timing changes between the detector pulse and the timing windows. This often resulted in shifts in the $2 \mathrm{D}$ histograms, requiring one to redraw the discriminating $2 \mathrm{D}$ gate. In addition, timing changes sometimes lowered the efficacy of the PSD electronics, allowing more accidental counts to pass than was usual. Accidental reduction was typically higher than a factor of 100 but was occasionally as low as a factor of 20. The PSD

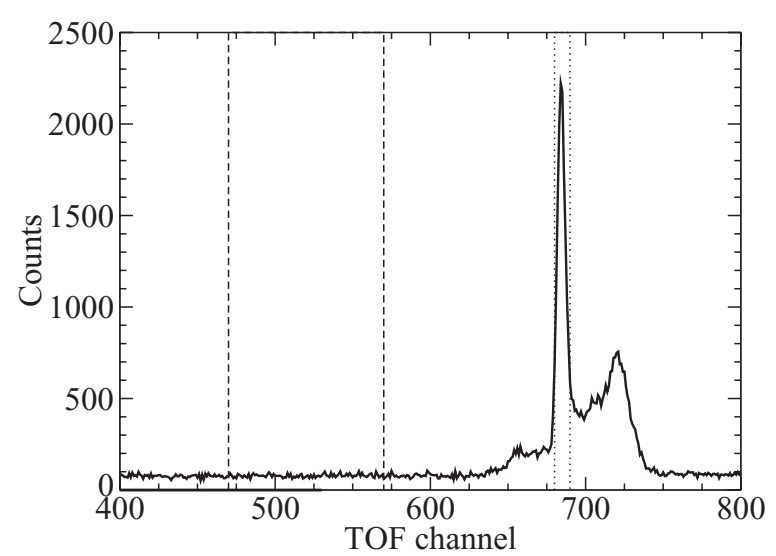

FIG. 3. TOF spectrum for $\theta_{\text {c.m. }}=136.2^{\circ}$ after gating with PSD. TOF increases to the right. A gate is shown for the elastic events (dotted vertical lines) and for the accidental coincidences (dashed).

of the polarimeter followed a more traditional method-a PSD module followed by a standard analog-to-digital converterand was relatively trouble free.

The 2D neutron gates in the PSD histograms were used to sort one-dimensional TOF spectra, which displayed the TOF between the $\mathrm{CD}$ and each side detector. Figure 3 displays a typical TOF spectrum after PSD filtering for the example $\theta_{\text {c.m. }}=136.2^{\circ}$. The $\gamma$ peak, once centered around channel 590 , has been entirely eliminated. The neutrons originating from the ground-state transition of the ${ }^{2} \mathrm{H}(\vec{d}, \vec{n})^{3} \mathrm{He}$ reaction and then undergoing $n$ - $d$ elastic scattering in the CD are represented by the large slender peak centered around channel 680. The wider peak blurring across the elastic peak from the right side is due to the "breakup neutrons" (neutrons originating from deuteron breakup reactions in the gas cell and in the $\mathrm{CD}$ ).

The PSD-filtered TOF spectra were, in turn, used to sort the center detector pulse height (CDPH) spectra. As shown in Fig. 3 two gates were used. The first cut (the two dotted vertical lines) was placed around the elastic counts (at approximately $25 \%$ of the peak height). The second cut (the two dashed vertical lines) was placed to the left of the $\gamma$ peak to determine the number of "accidental" counts. The accidental events are due to random coincidences between the center and side detectors and therefore are flat across the TOF spectrum. Because our electronics and PSD filtering usually reduced the accidental count rate to a low level, we made the width of the accidental window ten times greater than that of the elastic window to improve the statistical sample.

The CDPH spectra were sorted according to which side detector was involved (the right or left detector of each angle pair). In addition, separate CDPH spectra were created for each angle for spin-up or spin-down events and for elastic or accidental events. This resulted in a total of eight spectra for each angle pair. Sample CDPH spectra for $\theta_{\text {c.m. }}=136.2^{\circ}$, left side, spin up are displayed in Fig. 4(a). The solid curve shows the spectrum resulting from sorting with the elastic TOF gate and the dotted curve shows the spectrum from sorting with the accidental gate (after being renormalized by a factor of 10 , in keeping with the different TOF gate widths). We chose to display this example because it is near the maximum of the 

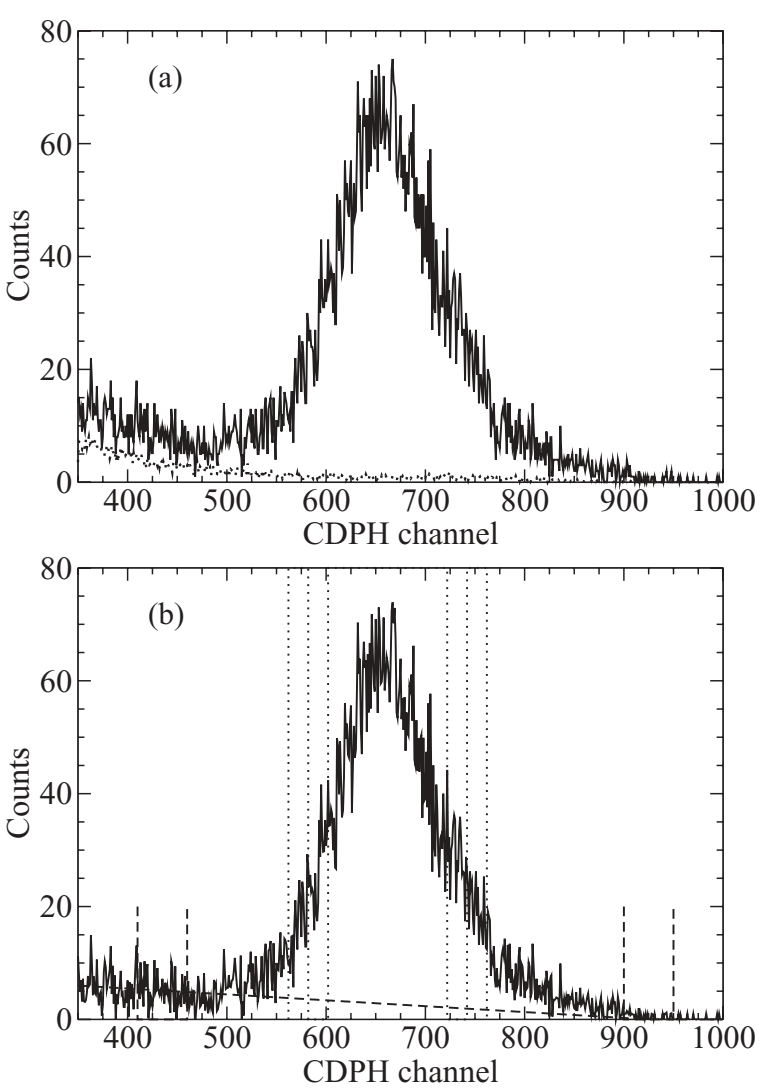

FIG. 4. CDPH spectra corresponding to the neutron side detector at $\theta_{\text {c.m. }}=136.2^{\circ}$. (a) The CDPH spectra after gating with the elastic TOF window (solid spectrum) and with the accidental TOF window (dotted spectrum). (b) The CDPH spectrum after the accidental background is subtracted. The dotted vertical lines are the three yield gates at 10,30, and 50\% of the CDPH peak. The dashed vertical lines are the windows used to define the linear fit background, which is shown here as the dashed slanted line.

present $A_{y}(\theta)$ angular distribution, which is near a minimum of the cross section and therefore subject to noise.

The accidental-gated CDPH spectra were now subtracted from the corresponding elastic-gated spectra. After subtraction a background was still visible on the left side of the elastic peak. We estimated the magnitude of the background by using two windows on the right and left of the peak and joining them by a straight line, which we refer to as the "fit background." In Fig. 4(b), the accidental-subtracted spectrum (in black) and the fit background (dashed slanted line) are displayed for $\theta_{\text {c.m. }}=$ $136.2^{\circ}$. The dotted vertical lines are the three gates used for the final yields, placed at approximately 10, 30, and $50 \%$ of the peak height.

In determining the final yields, the 10, 30, and 50\% yield windows all produced $A_{y}(\theta)$ results that were easily consistent with each other (within half of the final uncertainties). We judged the $30 \%$ window to afford the most prudent balance between maximizing yields and avoiding undesirable counts. In addition, the $30 \%$ gate was always close to the values suggested by gate scans. Therefore, we used the $30 \%$ window in calculating our final results. Within this gate the fit background varied from as low as $3 \%$ of the total counts at

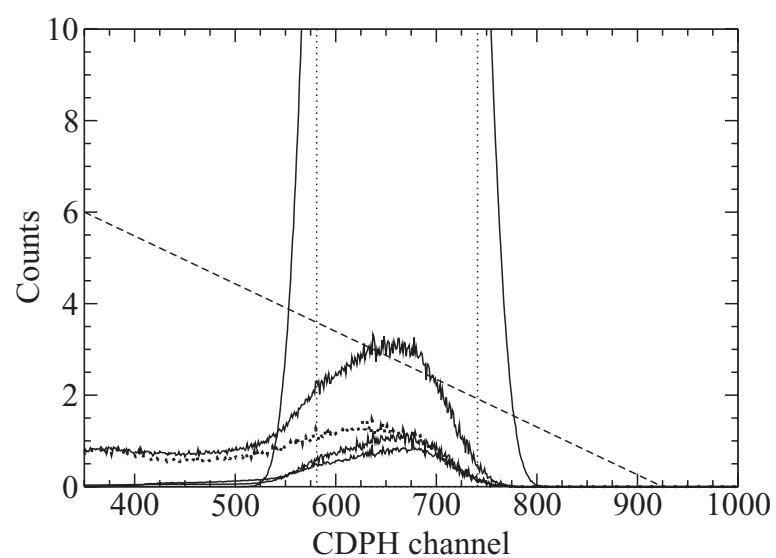

FIG. 5. CDPH spectrum for $\theta_{\text {c.m. }}=136.2^{\circ}$ showing the simulation results for multiple scattering. The fit background (dashed line) is the same as that of Fig. 4. Below this are the $d-{ }^{12} \mathrm{C}$ and ${ }^{12} \mathrm{C}-d$ events (the lowest two solid spectra), the $d$ - $d$ events (the dotted spectrum), and the total double-scattering events (solid). The large feature going off scale is the elastic peak and the dotted vertical lines are the $30 \%$ gate used in taking the final yields.

the forward angles to as high as $9 \%$ at the back angles. For the example of Fig. 4 this was $5.9 \%$.

\section{MONTE CARLO SIMULATION}

Part of the fit background is due to multiple scattering in the target. We determined the magnitude and the asymmetry of the multiple-scattering background by using a Monte Carlo computer simulation of neutron scattering in the target. Because the liquid deuterated scintillator NE232 is composed of $\mathrm{C}_{6}{ }^{2} \mathrm{H}_{12}$, we included three types of neutron double-scattering events in the simulation: $d-d, d-{ }^{12} \mathrm{C}$, and ${ }^{12} \mathrm{C}-d$.

Figure 5 displays an example of the Monte Carlo simulation results, normalized to the $\mathrm{CDPH}$ spectrum displayed in Fig. 4(b) for our example of $\theta_{\text {c.m. }}=136.2^{\circ}$. The large feature that goes off scale is the elastic peak, as reproduced by the simulation, while the dotted vertical lines are the $30 \%$ gate used for the final yields. The linear fit background is the dashed slanted line (the same as the background in Fig. 4). Below this one can see the contributions due to the $d-{ }^{12} \mathrm{C}$ and ${ }^{12} \mathrm{C}-d$ events (the lowest two solid spectra) and the $d-d$ events (dotted). The solid spectrum that intersects with the fit background represents the total double-scattering events.

The results for the ratio of the doubles counts to total counts $R_{\mathrm{ms}}$ and for the analyzing power $A_{y \mathrm{~ms}}$ for all three double-scattering processes combined are listed in Table II. Note that $R_{\mathrm{ms}}$ increases at the middle to back angles, largely because the cross section of $n-d$ scattering is lower. We stress the fact that the ratios were adjusted to the total number of experimental counts rather than simply the addition of the singles and doubles (which is all that is calculated by the simulation). We list only nominal values for $R_{\mathrm{ms}}$ to two significant figures since variations in residual background from batch to batch result in small variations of $R_{\mathrm{ms}}$. 
TABLE II. Monte Carlo simulation results of all three double-scattering processes in the target combined. For each center-of-mass angle, we show the percentage of total counts appearing in the $30 \%$ yield gate and the analyzing power.

\begin{tabular}{lcr}
\hline \hline$\theta_{\text {c.m. }}($ deg. $)$ & $R_{\mathrm{ms}}(\%)$ & \multicolumn{1}{c}{$A_{\text {yms }}(\theta)$} \\
\hline 46.7 & 1.5 & $0.0140 \pm 0.0021$ \\
57.4 & 1.6 & $0.0151 \pm 0.0023$ \\
67.9 & 1.9 & $0.0092 \pm 0.0070$ \\
78.0 & 2.2 & $0.0033 \pm 0.0047$ \\
87.7 & 2.4 & $-0.0176 \pm 0.0044$ \\
96.9 & 2.7 & $-0.0287 \pm 0.0040$ \\
101.1 & 2.6 & $-0.0223 \pm 0.0036$ \\
105.7 & 3.7 & $-0.0274 \pm 0.0017$ \\
113.9 & 4.4 & $0.0033 \pm 0.0029$ \\
119.1 & 4.6 & $0.0393 \pm 0.0034$ \\
124.6 & 5.5 & $0.0987 \pm 0.0039$ \\
131.5 & 5.2 & $0.1460 \pm 0.0021$ \\
136.2 & 5.2 & $0.1511 \pm 0.0024$ \\
141.0 & 4.6 & $0.1440 \pm 0.0020$ \\
146.6 & 4.3 & $0.1240 \pm 0.0021$ \\
152.0 & 4.5 & $0.0998 \pm 0.0031$ \\
\hline \hline
\end{tabular}

Note also that the doubles analyzing power $A_{y \mathrm{~ms}}$, given in Table II, somewhat mirrors the distribution of $n-d A_{y}(\theta)$ with a negative minimum around $100^{\circ}$ and a positive maximum around $130^{\circ}$. We confirmed that this is a reasonable result in a series of special tests on the simulation code; limitations due to kinematics and especially the TOF windows greatly limit which counts show up in the CDPH yield gates.

We also used the Monte Carlo simulation to determine the number of so-called "edge effect deuterons." These are recoil deuterons that hit the edge of the $\mathrm{CD}$ before depositing their full energy in the scintillator. The edge-effect events appeared as a flat shoulder on the left side of the elastic peak and accounted for only a small part of the fit background. The ratio of edgeeffect counts to total counts $R_{\mathrm{ee}}$ ranged from about $0.1 \%$ of the total counts at the forward angles to $0.6 \%$ at the back angles. Of course, the analyzing power of the edge-effect events $A_{\text {yee }}$ is virtually identical to that of the normal elastic events.

Because the library of the Monte Carlo code must include $n$ - $d A_{y}(\theta)$ at $19.0 \mathrm{MeV}$, we initially placed raw experimental data into the library to perform the first wave of calculations. After applying the resulting corrections to our data (as described in the following), we then performed a "self-consistency check" by placing the corrected $n$ - $d$ data into the library and re-running the simulation. The analyzing power values for multiple scattering and edge effects changed slightly as a result of the self-consistency check. The effect of the second wave of calculations on the count ratios and on the $n-d A_{y}(\theta)$ data itself was insignificant.

The multiple scattering and edge-effect events were now removed from the fit background, leaving a "residual background." A common way of removing these events is to normalize the Monte Carlo simulation to the experiment and then to subtract the simulated multiple-scattering and edge-effect spectra from the experimental CDPH spectra. This method was not followed in the current analysis. This is because changes of beam polarization and CDPH gain limited our ability to combine experimental batches, greatly increasing the number of Monte Carlo spectra that had to be normalized.

Instead we turned to a method that works equally well when applied with care. This method starts with the experimental yields and analyzing powers after accidental subtraction, at the stage represented by Fig. 4(b), as well as the simulation results for multiple scattering and edge effects. It also uses a familiar relation that combines the analyzing powers of different scattering processes. Our analysis used two such relations. One is based on the known experimental analyzing power of the accidental-subtracted data

$$
R_{\text {acc }} A_{y \text { acc }}=R_{\mathrm{el}} A_{\text {yel }}+R_{\mathrm{ms}} A_{y \mathrm{~ms}}+R_{\mathrm{ee}} A_{\text {yee }}+R_{\mathrm{res}} A_{\text {yres }},
$$

where the $R$ are the ratios for each scattering process relative to the total counts and where the subscripts acc, el, $m s$, $e e$, and res stand for accidental subtracted, elastic, multiple scattering, edge effect, and residual background, respectively. Information on the left side of the equation is known from the experiment. Depending on the measured angle and the run, the percentage of accidental counts ranged from about 0.5 to $5 \%$. Therefore, $R_{\text {acc }}$ varied from about 99.5 to $95 \%$. Information of the multiple-scattering and edge effects is known from the Monte Carlo code. As was mentioned previously, the ratios from the simulation were adjusted downward, in accordance with the total number of counts in the experiment (which contains the residual background that the code cannot simulate).

A second relation is based on the known experimental analyzing power of the fit background

$$
R_{\mathrm{fit}} A_{y \mathrm{fit}}=R_{\mathrm{msfit}} A_{y \mathrm{~ms}}+R_{\mathrm{ee}} A_{y \mathrm{ee}}+R_{\mathrm{res}} A_{y \mathrm{res}},
$$

where fit refers to the fit background. The ratio of multiplescattering counts accounted for by the fit background $R_{\mathrm{msfit}}$ appearing in Eq. (4) is different from the $R_{\mathrm{ms}}$ of Eq. (3). As is discussed in the following, the reason for this difference is that the shape of the doubles-counts CDPH spectra are not always flat (as they are at the forward angles) but sometimes have a peak inside the yields gates (at the back angles), as is visible in Fig. 5.

The objective of our "ratio method" is to compute the four unknowns $R_{\text {res }}, A_{\text {yres }}, R_{\mathrm{el}}$, and $A_{\text {yel }}$. Two further relations involving only the ratios make this possible. The first asserts that all of the counts in the yields gate must add to the accidental-subtracted values, $R_{\mathrm{acc}}=R_{\mathrm{el}}+R_{\mathrm{ms}}+R_{\mathrm{ee}}+R_{\text {res }}$. The second involves the counts composing the fit background, $R_{\mathrm{fit}}=R_{\mathrm{msfit}}+R_{\mathrm{ee}}+R_{\mathrm{res}}$.

To exemplify the difference between the doubles counts appearing in the yields gates $\left(R_{\mathrm{ms}}\right)$ and the doubles counts accounted for by the fit background $\left(R_{\mathrm{msfit}}\right)$, return to the example of $\theta_{\text {c.m. }}=136.2^{\circ}$. The quantity $R_{\mathrm{ms}}$ was found by finding the ratio of doubles to total counts inside the yields gates. Meanwhile, the quantity $R_{\mathrm{msfit}}$ was found by using the background windows in Fig. 4(b) to draw a new linear background resting on the left shoulder of the total doublescattering spectrum of Fig. 5. Of course, this new background was considerably lower than the fit background. The number of counts of this new background was found inside the yields 
TABLE III. Final $n$ - $d$ analyzing power data at $E_{n}=19.0 \mathrm{MeV}$

\begin{tabular}{lr}
\hline \hline$\theta_{\text {c.m. }}($ deg. $)$ & \multicolumn{1}{c}{$A_{y}(\theta)$} \\
\hline 46.7 & $0.0356 \pm 0.0023$ \\
57.4 & $0.0339 \pm 0.0026$ \\
67.9 & $0.0236 \pm 0.0022$ \\
78.0 & $0.0079 \pm 0.0033$ \\
87.7 & $-0.0109 \pm 0.0028$ \\
96.9 & $-0.0292 \pm 0.0037$ \\
101.1 & $-0.0352 \pm 0.0052$ \\
105.7 & $-0.0302 \pm 0.0046$ \\
113.9 & $-0.0045 \pm 0.0061$ \\
119.1 & $0.0415 \pm 0.0089$ \\
124.6 & $0.1164 \pm 0.0102$ \\
131.5 & $0.2309 \pm 0.0132$ \\
136.2 & $0.2031 \pm 0.0129$ \\
141.0 & $0.1581 \pm 0.0092$ \\
146.6 & $0.1114 \pm 0.0072$ \\
152.0 & $0.0817 \pm 0.0064$ \\
\hline \hline
\end{tabular}

gates and then its ratio was taken with the singles counts. Once again, this was adjusted down to reflect the total number of counts found in the experimental spectra, giving $R_{\mathrm{msfit}}$.

Because $\theta_{\text {c.m. }}=136.2^{\circ}$ is a back angle, the percentage of counts inside the $30 \%$ yield window due to the fit background was relatively high: $R_{\mathrm{fit}}$ was $5.9 \%$ of the total counts. As suggested by the doubles spectrum of Fig. 5 (the peak of which goes above the fit background around channel 670), the percentage of double-scattering counts was quite similar: $R_{\mathrm{ms}}$ was $5.2 \%$. However, due to the relatively low left shoulder for this spectrum, double scattering accounted for less than one quarter of the fit background: $R_{\mathrm{msfit}}$ was $1.2 \%$.

The analysis described in this section yielded the four quantities $R_{\mathrm{el}}, A_{\text {yel }}, R_{\text {res }}$, and $A_{\text {yres }}$. The ratio of elastic events to total counts $R_{\mathrm{el}}$ varied from about $96 \%$ at the more forward angles to $90 \%$ at the back angles. The $A_{y \text { el }}$ values are the final $n-d A_{y}(\theta)$ data reported in Table III. Before presenting the final data, however, we discuss our treatment of the background that remained after the subtraction of multiple scattering and edge effects from the fit background.

\section{RESIDUAL BACKGROUND}

Our treatment of the residual background started from the quantities $R_{\text {res }}$ and $A_{y \text { res }}$ computed in the previous section. Our determination of the linear fit background and subtraction of multiple-scattering and edge-effect counts led to a reliable determination of $R_{\text {res }}$, the ratio of the residual-background counts to total counts (inside the $30 \%$ yield gate). This was relatively small for the forward angles, as low as $1 \%$, but was larger for the back angles, as high as 7\%. The major consequence of considering the difference between $R_{\mathrm{ms}}$ and $R_{\mathrm{msfit}}$ was to increase the residual background at the back angles.

However, our statistics were not good enough to determine reliably the analyzing power of the residual background $A_{y \text { res }}$. This was due partly to the fact that the left shoulder of the CDPH elastic peak was not well defined to begin with. Careful sorting reduced the residual background to less than $10 \%$ of an already low-counting experiment; the spectrum of Fig. 4 is based on nearly four days of beam time. Another reason was that we can only sample a portion of the left shoulder of the CDPH peak due to a slight curvature of the shoulder. Attempts at departing from the linear background and using second-order polynomials proved to be even more sensitive to statistical variations.

Despite the limitation of the statistical sample, the residual background was relatively easy to account for. We did three different calculations of the final analyzing power values $A_{y \mathrm{el}}$. In the first calculation, we took whatever the analysis of the previous section gave us for the analyzing power of the residual background $A_{\text {yres. }}$. In the second calculation we assumed that the residual background had an asymmetry of zero; that is, we set $A_{\text {yres }}$ equal to zero. In a third calculation, we assumed that the residual background had the same analyzing power as that of the elastic peak; that is, we set $A_{y \text { res }}$ equal to $A_{y \mathrm{el}}$.

None of the three calculation methods made significant differences to the final $A_{y \text { el }}$ values, as is demonstrated in Fig. 6. The figure is broken up into two panels for two angle regimes in the interest of readability. The $A_{y}(\theta)$ data using the polarized residual background are shown as the crosses. The final data using an unpolarized residual background are shown as the
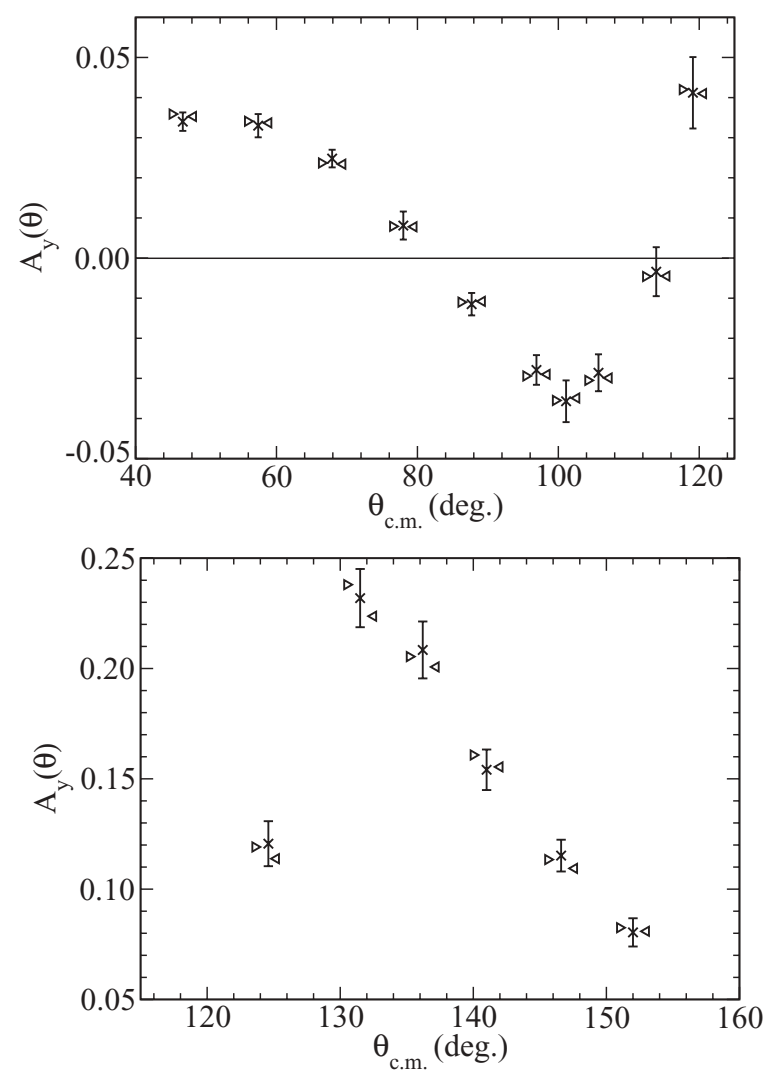

FIG. 6. $A_{y}(\theta)$ data using the three treatments of the residual background: polarized (the crosses with uncertainties), unpolarized (the triangles offset to the left), and with the same polarization as the elastic counts (the triangles offset to the right). The final data listed in Table III and Fig. 8 averages the unpolarized and same-polarization results. See text for details. 
triangles offset about $1^{\circ}$ to the left, while the final data using the same-polarization assumption are the triangles offset about $1^{\circ}$ to the right. The uncertainties are associated with the crosses but are suppressed for the other two sets of results in the interest of clarity.

This pleasing result suggested a solution: to assign the residual background an asymmetry between zero and that of the elastic counts. As Fig. 6 shows, the $A_{y \text { el }}$ results using a polarized residual background are consistent, within uncertainties, with the other two sets of results. Although there is some fluctuation in the results using a polarized background, these fluctuations are small and do not favor either of the other sets.

Two other pieces of evidence lend credence to the idea that the residual background has an asymmetry somewhere between zero and that of the elastic counts. First, one of the likely components of the residual background is the breakup neutrons, a small amount of which we were forced to include in our elastic TOF gate (see Fig. 3). In the CDPH spectrum, these counts are mostly separated from the elastic peak but tail in somewhat from the left (low-energy) side. From our experience with the polarimeter, we know that the breakup events have an asymmetry that is extremely close to that of the elastic counts.

Second, we performed "gate scans" in which a relatively narrow yield gate is stepped across each CDPH spectrum, from the low-energy left shoulder (which carries most of the background) to the high-energy shoulder (which has very few counts). For each placement of the narrow gate, the asymmetry is calculated using Eq. (1). We found that variations of the asymmetry of the left shoulder were less than those of the fit background and the residual background, both of which rely on the drawing of linear backgrounds. Also, we found that the nominal asymmetry of the left shoulder did not deviate greatly from the range between zero and that of the elastic counts. An example is displayed in Fig. 7, once again for $\theta_{\text {c.m. }}=136.2^{\circ}$. Note that the left background window rests between channels 410 and 460 (this appears in Fig. 4(b) but is dropped here).

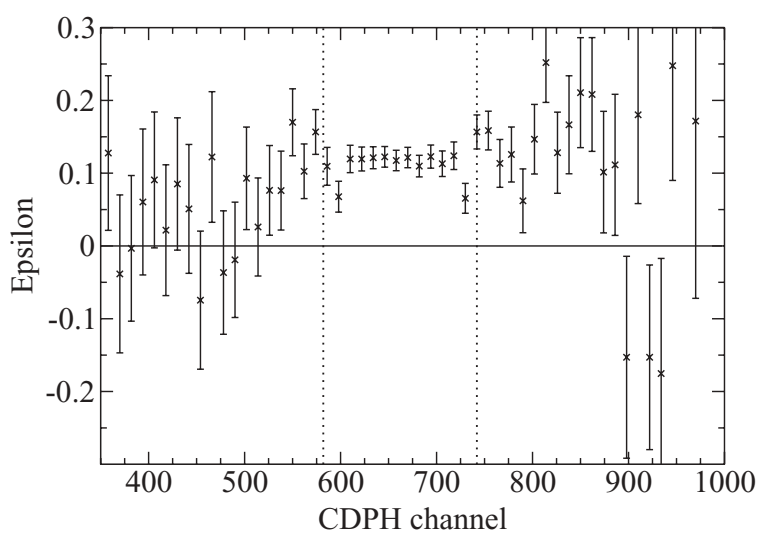

FIG. 7. Gate scan showing the asymmetry, computed with Eq. (1), across the CDPH spectrum for $\theta_{\text {c.m. }}=136.2^{\circ}$. The $30 \%$ gate used for the final yields, shown as the dotted vertical lines, is 160 channels wide. The width of the gate used for the scan was 12 channels.
In light of the previous considerations, we defined the final $n-d A_{y}(\theta)$ results as the average between the treatment using the unpolarized residual background and the treatment using the residual background with the same polarization as the elastic counts (the left and right triangles, respectively, of Fig. 6). As an estimate of the uncertainty associated with this treatment of the residual background, we took half of the difference between the two analyzing power results. We then added this linearly to the uncertainties of the final $A_{y}(\theta)$ data.

\section{FINAL RESULTS AND DISCUSSION}

The final $n-d A_{y}(\theta)$ data at $E_{n}=19.0 \mathrm{MeV}$ are listed in Table III. The effective angle is listed as calculated by the Monte Carlo simulation to account for finite-geometry effects. The uncertainties of the final $A_{y}(\theta)$ data include five contributions, all of which were discussed previously. In addition to the statistical uncertainties, we included the uncertainties associated with the removal of multiple-scattering and edge effects from the fit background. An uncertainty of $1.7 \%$ was applied to our determination of the beam polarization due to the uncertainties of the phase shift analysis [26] and our multiple-scattering corrections. For the first two of our five ten-day runs, we added an additional $1.5 \%$ uncertainty to the determination of the polarimeter's asymmetry due to the fact that we monitored the asymmetry of the breakup neutrons and then translated this to the asymmetry of the elastic neutrons. We combined these four uncertainties in quadrature. The fifth uncertainty due to our treatment of the residual background was added linearly.

The final $n$-d $A_{y}(\theta)$ data are also displayed in Fig. 8, where it is compared to three-body calculations based on the CD-Bonn $N N$ potential model (solid curve). A Legendre polynomial fit (dashed curve) is included as a guide to the eye. The relative difference between theory and experiment, measured in the manner of Ref. [24], by taking account of both the maximum and the minimum of the angular distribution is $21 \% \pm 3 \%$. This is somewhat lower than the $25 \%$ discrepancy found in most of the data from 1.2 to $22.7 \mathrm{MeV}$. It is

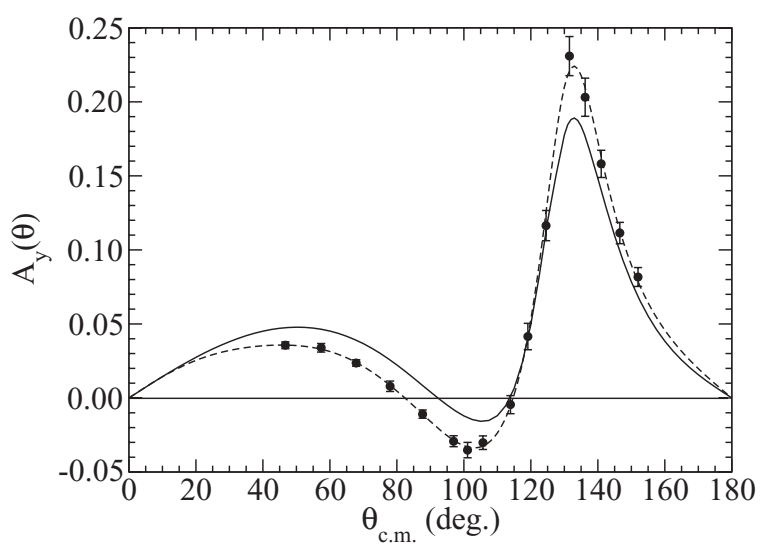

FIG. 8. Final $n$ - $d$ analyzing power data at $19.0 \mathrm{MeV}$ compared to a prediction of a three-body Faddeev calculation (solid curve). The Legendre polynomial fit (dashed curve) is included to guide the eye through the data. 
considerably lower than the relative difference of $36 \%$ given by $n-d A_{y}(\theta)$ data at $16 \mathrm{MeV}$ [15] and $33 \%$ by $p$ - $d$ data at $20.15 \mathrm{MeV}$ [24]. We place greater trust in our present data (and other $p$ - $d$ data at 16,18 , and $22.7 \mathrm{MeV}$ ), which are of higher quality and precision than the two data sets just mentioned.

As noted in the Introduction, the present angular distribution at $19.0 \mathrm{MeV}$ is only a start at addressing the lack of $n$-d $A_{y}(\theta)$ data above $16 \mathrm{MeV}$. Taking data between 20 and $30 \mathrm{MeV}$ is desirable but must wait for a nuclear facility with both a polarized-ion source and a relatively high-energy neutron source. While TUNL has an excellent ABPIS source, the present study is near the top edge of the facility's energy capability. Although we are currently at work on $n-d A_{y}(\theta)$ data at 21.0 and $22.5 \mathrm{MeV}$, we cannot continue to higher energies unless we make use of a tritiated target. This is unlikely given current federal regulations concerning the procurement of nuclear materials.

Of course, the solution of the 3NAPP depends not only on new data but also on new theoretical developments. As discussed in the Introduction, we currently await calculations at next-to-next-to-next-to leading order (N3LO) in chiral perturbation theory, which have the promise of including some relatively long-range $3 \mathrm{NF}$ effects. As existing $n-d$ and $p-d A_{y}(\theta)$ data showed, explaining the 3 NAPP will require accounting for a relative difference between experiment and theory that is nearly flat at $25 \%$ from 1.2 to $16 \mathrm{MeV}$. The present data suggest that this discrepancy is reduced somewhat at 19.0 MeV (although determining the exact energy dependence must wait for further data). In any case, the existence of such a large discrepancy over such a large range of energy suggests that there may be more than one cause of the 3NAPP, perhaps involving deficiencies in the two-body force as well as our current neglect of certain $3 \mathrm{NF}$ effects.

\section{ACKNOWLEDGMENTS}

This work was supported in part by the US Department of Energy, under Grant No. DE-FG02-97ER41033. We acknowledge the help of Penn State Altoona student Bradley D. Buck and NSF-Duke REU student Patrick D. Ulrich. We also thank Steve Churchwell for helping with the experiment and Shigeyuki Tajima for the 2D gate software, which improved our data analysis.
[1] V. G. J. Stoks, R. A. M. Klomp, C. P. F. Terheggen, and J. J. de Swart, Phys. Rev. C 49, 2950 (1994).

[2] R. B. Wiringa, V. G. J. Stoks, and R. Schiavilla, Phys. Rev. C 51, 38 (1995).

[3] R. Machleidt, Phys. Rev. C 63, 024001 (2001).

[4] H. Witała, W. Glöckle, J. Golak, A. Nogga, H. Kamada, R. Skibiński, and J. Kuroś-Zolnierczuk, Phys. Rev. C 63, 024007 (2001).

[5] J. Kuroś-Zolnierczuk, H. Witała, J. Golak, H. Kamada, A. Nogga, R. Skibiński, and W. Glöckle, Phys. Rev. C 66, 024003 (2002).

[6] H. Witała, D. Hüber, and W. Glöckle, Phys. Rev. C 49, R14 (1994).

[7] E. M. Neidel, W. Tornow, D. E. Gonzalez-Trotter, C. R. Howell, A. S. Crowell, R. A. Macri, R. L. Walter, G. J. Weisel, R. S. Pedroni, D. M. Markoff, and H. Witała, Phys. Lett. B552, 29 (2003).

[8] J. E. McAninch, L. O. Lamm, and W. Haeberli, Phys. Rev. C 50, 589 (1994).

[9] W. Tornow, C. R. Howell, M. Alohali, Z. P. Chen, P. D. Felsher, J. M. Hanly, R. L. Walter, G. Weisel, G. Mertens, I. Slaus, H. Witała, and W. Glöckle, Phys. Lett. B257, 273 (1991).

[10] W. Tornow et al., Phys. Lett. B203, 341 (1988).

[11] W. Tornow, C. R. Howell, R. C. Byrd, R. S. Pedroni, and R. L. Walter, Phys. Rev. Lett. 49, 312 (1982).

[12] C. R. Howell, W. Tornow, K. Murphy, H. G. Pfutzner, M. L. Roberts, Anli Li, P. D. Felsher, R. L. Walter, P. A. Treado, and Y. Koike, Few-Body Syst. 2, 19 (1987); N. Nishimori et al., Nucl. Phys. A631, 697c (1998).
[13] J. Cub et al., Few-Body Syst. 6, 151 (1989).

[14] W. Tornow, R. C. Byrd, C. R. Howell, R. S. Pedroni, and R. L. Walter, Phys. Rev. C 27, 2439 (1983).

[15] T. Fujita, K. Sagara, K. Shigenaga, K. Tsuruta, T. Yagita, T. Nakashima, N. Nishimori, H. Nakamura, and H. Akiyoshi, AIP Conf. Proc. 570, 709 (2001); K. Sagara (private communication).

[16] H. Dobiasch, R. Fisher, B. Haesner, H. O. Klages, P. Schwarz, B. Zeitnitz, R. Maschuw, K. Sinram, and K. Wick, Phys. Lett. B76, 195 (1978).

[17] H. Witała, J. Golak, R. Skibiński, C. R. Howell, and W. Tornow, Phys. Rev. C 67, 064002 (2003).

[18] A. Kievsky, M. Viviani, and L. E. Marcucci, Phys. Rev. C 69, 014002 (2004).

[19] W. Tornow, H. Witała, and A. Kievsky, Phys. Rev. C 57, 555 (1998).

[20] E. Epelbaum, H. Kamada, A. Nogga, H. Witała, W. Glöckle, and Ulf-G. Meissner, Phys. Rev. Lett. 86, 4787 (2001).

[21] H. Witała, J. Golak, W. Glöckle, and H. Kamada, Phys. Rev. C 71, 054001 (2005).

[22] E. Epelbaum, A. Nogga, W. Glöckle, H. Kamada, Ulf-G. Meissner, and H. Witała, Eur. Phys. J. A 15, 543 (2002).

[23] E. Epelbaum, A. Nogga, W. Glöckle, H. Kamada, Ulf-G. Meissner, and H. Witała, Phys. Rev. C 66, 064001 (2002).

[24] W. Tornow, J. H. Esterline, and G. J. Weisel, J. Phys. G 35, 1 (2008).

[25] J. Zamudio-Cristi, B. E. Bonner, F. P. Brady, J. A. Jungerman, and J. Wang, Phys. Rev. Lett. 31, 1009 (1973).

[26] Th. Stammbach and R. L. Walter, Nucl. Phys. A180, 225 (1972). 